\title{
ENHANCEMENT IN PLANT WATER RELATIONS AND FATTY ACID PROFILE IN SUNFLOWER (HELIANTHUS ANNUUS L.) THROUGH APPLICATION OF ABSCISIC ACID UNDER VARIED WATER LEVELS
}

\author{
SHAH, T. ${ }^{1,2}-$ KHAN, H. ${ }^{1}-$ NOOR, M. A. ${ }^{3 *}-$ WANG, X. ${ }^{4 *}-$ BASAHI, M. A. ${ }^{5}-$ NASIR, M. ${ }^{6}$ \\ ${ }^{I}$ Department of Agronomy, University of Agriculture, Peshawar, Pakistan \\ ${ }^{2}$ Key Laboratory of Oil Crop Biology and Genetic Improvement of the Ministry of Agriculture, \\ Oil Crops Research Institute of the Chinese Academy of Agricultural Sciences, Wuhan, Hubei, \\ China \\ ${ }^{3}$ Institute of Crop Sciences, Chinese Academy of Agricultural Sciences, Key Laboratory of Crop \\ Physiology and Ecology, Ministry of Agriculture, 100081 Beijing, China \\ ${ }^{4}$ College of Life Sciences, Yan'an University, 716000 Yan'an, Shaanxi, China \\ ${ }^{5}$ Dept. of Biology, College of Science \& Arts, Sajir, Shaqra University \\ P.O. Box-33, 11961 Shaqra, Saudi Arabia \\ ${ }^{6}$ College of Natural Resources and Environment, Northwest Agriculture and Forestry \\ University, 712100 Yangling, China \\ *Corresponding author \\ e-mail:mehmood2017@gmail.com; wangxiukang@126.com \\ (Received $9^{\text {th }}$ May 2018; accepted $17^{\text {th }}$ Jul 2018)
}

\begin{abstract}
Enhancement in yield and quality of sunflower by foliar spray of abscisic acid (ABA) under water deficit condition was studied during 2016 and 2017. Three irrigation treatments were applied. Four irrigations (at 4-6 leaf, at vegetative, at flowering and at grain formation stage), three irrigations (at 4-6 leaf, at pre flowering and at grain formation stage), three irrigations (at 4-6 leaf, at vegetative and at grain formation stage). Sunflower hybrid (S-278) was sprayed with different ABA concentrations $(0,10 \mu \mathrm{m}$ and $20 \mu \mathrm{m})$ at 4-6 leaf, vegetative, flowering and grain formation stage. Foliar application of $10 \mu \mathrm{M}$ ABA under water stress at vegetative stage significantly enhanced plant height, head diameter, achene yield and biological yield. It also enhanced sunflower water relations by increasing water potential. ABA application and water deficit showed opposite results for yield and oil quality. Water deficit at vegetative or at flowering stage maximized stearic and oleic acid contents and minimized palmitic and linoleic acid contents, whereas foliar spray of ABA under water stress at both stages reduced stearic and oleic acid but maximized palmitic and linoleic acid.
\end{abstract}

Keywords: abscisic acid, relative water contents, turgor pressure, oleic acid, palmitic acid, linoleic acid, sunflower

\section{Introduction}

Drought is the foremost yield-limiting feature for sunflower (Helianthus annuus L.) productivity in semi-arid areas (Rauf et al., 2015; Daryanto et al., 2016). In different parts of the world oil and achene yield losses due to drought stress were described (Woli et al., 2014; Yin et al., 2014). Water scarcity encourages significant alterations in physiological and biochemical processes implicated in biomass production, and alters dry matter partitioning (Fernández et al., 2012; Wu et al., 2017). Breeding for drought tolerance is thus indispensable to diminish yield losses in sunflower in drought-prone 
regions (Rauf et al., 2015). In order to recognize probable sources of drought tolerance, cultivated sunflower breeding lines from different sources were assessed on the basis of comparative performance under drought stress (Hussain et al., 2017). Nevertheless, the narrow genetic base of breeding lines was one of the major restrictions for the possibility of such studies.

Several studies showed, abiotic stresses activate several biochemical, physiological and molecular responses that effect several plant processes at cellular level (Wang et al., 2003; Hasanuzzaman et al., 2013; Tsironi and Taoukis, 2017). To fight several environmental pressures, dynamic methods and techniques should be developed (Yin et al., 2017). Among various control measures and monitoring tools (remote sensing) to assess the impacts of drought stress on crop plants (Villegas et al., 2017; Natsagdorj et al., 2017; Song et al., 2017), agronomic measures for water conservation and chemical plant growth regulators are of great interest (Ahmad et al., 2016). Phytohormone engineering could be considered as a preferable technique to increase the production. Phytohormones are the substantial regulators in plant growth and development and also intermediaries of environmental stress responses (Sreenivasulu et al., 2012). Among several phytohormones, abscisic acid is the vital regulator of abiotic stress resistance in plants and synchronizes an arrangement of roles (Finkelstein, 2013; Wani and Kumar, 2015), allowing plants to survive with diverse stresses. In the plant, the level of abscisic acid increases through abscisic acid biosynthesis when environmental conditions are harsh. The augmented abscisic acid binds to its receptor to start signal transduction causing cellular responses to different stresses ( $\mathrm{Ng}$ et al., 2014); thus, ABA is also termed a stress hormone (Mehrotra et al., 2014). ABA was firstly suggested to be implicated in abscission and has later been revealed to play a role in plant growth and development, comprising cell division followed by elongation, embryo maturation, seed dormancy, germination, root growth, floral initiation, and responses to both biotic and abiotic stresses, such as osmotic stress, chilling, high salinity, drought, pathogen attack and UV radiation (Finkelstein, 2013; Yoshida et al., 2014; Sah et al., 2016). ABA is significantly increased under drought or salinity stress conditions, stimulating stomatal closure, change in gene expression, and adaptive physiological responses (Kim et al., 2010; Sah et al., 2016). Abscisic acid also plays a vital role in many processes at cell level including dormancy, seed development, vegetative growth, germination etc. (Finkelstein et al., 2013; Saradadevi et al., 2017) and variation in root morphology (Harris, 2015). Meanwhile the discovery of abscisic acid, numerous struggles have been dedicated to understanding how abscisic acid is produced under stress conditions. In stressful conditions like extreme temperature, drought, and high salinity, content in plants enhances significantly, stimulating stress-tolerance effects that support plants, acclimatize, and endure under these stressful conditions ( $\mathrm{Ng}$ et al., 2014). Under nonstress situations, abscisic acid is also mandatory for plant overall growth and development.

Around the globe enormous research has been done on various crop plants to enhance water use efficiency (WUE) by sowing better varieties and improvement of drought tolerance by foliar application of abscisic acid (ABA). But little or no data is existing on the interaction of abscisic acid and use of diverse cultivars of Helianthus annuus to alleviate the impacts of water stress in different agro-ecological conditions of Pakistan. Therefore the current study has been planned to adjust ABA application stage for attaining greater yields of sunflower under water stress and to associate the performance of different Helianthus annuus hybrids under water stress environments. 


\section{Materials and methods}

\section{Study area, soil and weather condition}

Field experiments were conducted to study the response of spring planted sunflower hybrids to different irrigation schedules and foliar spray of abscisic acid. Trials were conducted in 2016 and 2017 at the Agronomic Research Farm, University of Agriculture, Peshawar, Pakistan. The climate of Peshawar region is semi-arid $\left(34.01^{\circ} \mathrm{N}\right.$, $71.35^{\circ} \mathrm{E}$ ) at an altitude of 350 meters above sea level. Peshawar is situated about $1600 \mathrm{~km}$ north of the Indian Ocean. All the analytical work associated to research was conducted in the Stress Physiology Laboratory, Nuclear Institute for Food and Agriculture, NIFA, Peshawar. Soil analysis of the experimental soil was given in Table 1. The climate of the area is semiarid where the mean annual rainfall is very low (300 to $500 \mathrm{~mm}$ ), 60-70\% rainfall occurs in summer, whereas the remaining $30-40 \%$ rainfall occurs in winter (Fig. 1).

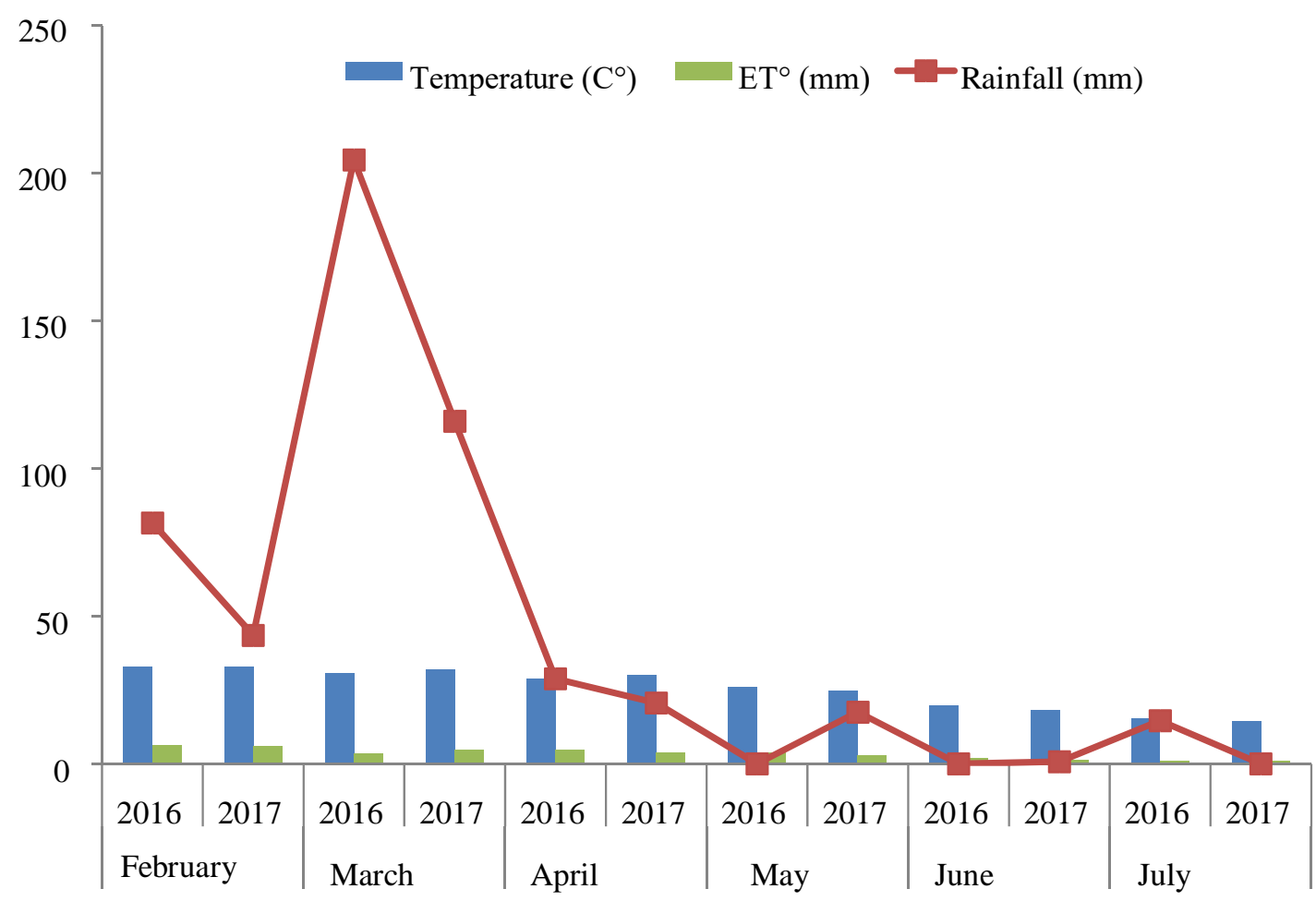

Figure 1. Monthly meteorological data for experimental years 2016 and 2017 of Agronomic Research Farm, University of Agriculture, Peshawar, Pakistan

\section{Experimental design and treatments}

The layout of experiment was randomized complete block design (RCBD) with factorial arrangement with three replications. Net plot size was $3.0 \mathrm{~m} \times 6.0 \mathrm{~m}$. Three irrigation schedules (I) were imposed which were $\left(\mathrm{I}_{1}\right)$ four irrigations (at 4-6 leaf, at vegetative, at flowering and at grain formation stage), $\left(\mathrm{I}_{2}\right)$ three irrigations (at 4-6 leaf, at pre flowering and at grain formation stage), $\left(\mathrm{I}_{3}\right)$ three irrigations (at 4-6 leaf, at vegetative and at grain formation stage $)$ and five $A B A$ concentration $\left(\mathrm{C}_{1}\right.$ : control, $\mathrm{C}_{2}$ : $10 \mu \mathrm{M}$ ABA at vegetative stage; $\mathrm{C}_{3}: 10 \mu \mathrm{M}$ ABA at flowering stage, $\mathrm{C}_{4}: 20 \mu \mathrm{M}$ ABA at 
vegetative stage and $\mathrm{C}_{5}: 20 \mu \mathrm{M} A B A$ at flowering stage. The first irrigation was applied when the plant had 4-6 leaves (25 DAS), the 2nd irrigation was applied at vegetative stage (45 DAS) excluding the plots, which were exposed to water deficit at this stage, the 3rd irrigation was applied at flowering stage (67 DAS) excluding the plots, which were exposed to water deficit at this stage. The 4th irrigation was given to all plots at grain formation stage (90 DAS). ABA was weighed according to per treatment and was added in a graduated cylinder and volume was made $1 \mathrm{~L}$ in volumetric flask with distilled water. Afterward Knapsack sprayer was calibrated $\left(250 \mathrm{~L} \mathrm{ha}^{-1}\right)$ and used to spray solution. While distilled water was sprayed on the control plots.

Table 1. Physicochemical analysis of soil during 2016-17

\begin{tabular}{c|c|c|c}
\hline \multirow{2}{*}{ Characteristic } & \multirow{2}{*}{ Unit } & \multicolumn{2}{|c}{ Value } \\
\cline { 3 - 4 } & & $\mathbf{2 0 1 6}$ & $\mathbf{2 0 1 7}$ \\
\hline Sand & $\%$ & 54 & 56 \\
Silt & $\%$ & 24.2 & 20 \\
Clay & $\%$ & 21.8 & 22 \\
Textural class & - & Sandy clay loam & Sandy clay loam \\
Saturation percentage & $\%$ & 32 & 30 \\
Ec & $\mathrm{dS} / \mathrm{m}$ & 1.44 & 1.56 \\
pH & - & 7.6 & 7.9 \\
Organic matter & $\%$ & 0.71 & 0.68 \\
Organic carbon & $\%$ & 0.44 & 0.39 \\
Total nitrogen & $\%$ & 0.041 & 0.038 \\
Available P & $\mathrm{ppm}$ & 6.1 & 6.6 \\
Available K & $\mathrm{ppm}$ & 135 & 131 \\
\hline
\end{tabular}

\section{Crop husbandry}

The seedbed was prepared by pre-soaking up to $10 \mathrm{~cm}$ irrigation then cultivating the field for 2-3 times with tractor-mounted cultivator. Helianthus annuus hybrids were sown on 19th and 13th of February 2016 and 2017, on ridges using dibbler with seed rate of $8 \mathrm{~kg} \mathrm{ha}^{-1}$. Ridges were made $75 \mathrm{~cm}$ distant and plant to plant distance of $25 \mathrm{~cm}$ was kept. Seed of sunflower hybrid S-278 were obtained from Syngenta Company. were obtained from Fertilizers were applied at the rate of $100 \mathrm{~kg} \mathrm{P}_{2} \mathrm{O}_{5} \mathrm{ha}^{-1}$ in the form of triple super phosphate (TSP) and $150 \mathrm{~kg} \mathrm{~N}$ in the form of urea. Half nitrogen and full phosphorus were applied at sowing, whereas remaining nitrogen was applied with 1 st irrigation. Irrigation was done as per treatment by flooding. To keep field free from weeds field hoeing was done. Plant protection measures were conducted to keep crop free of insect pests, diseases and parrots. For control of whitefly and head rot Polo and Radomil Gold were applied, respectively. The crop was harvested on May 30, 2016 and June 6, 2017.

\section{Measurements}

\section{Agronomic traits}

From each plot, ten plants were selected randomly. The plant height was determined with measuring tape and then averaged. In order to find head diameter, 10 heads were 
randomly selected from each plot and their diameter was measured with the help of a measuring tape and then averaged. For calculation of achene yield plants were harvested at maturity, sunflower' heads were detached after drying then threshed manually to find the achene yield plot $^{-1}$. The moisture contents were calculated from random achene samples. The achene yield was attuned to $10 \%$ moisture content and expressed in $\mathrm{kg} \mathrm{ha}^{-}$ ${ }^{1}$. Weight of air-dried plants (excluding achenes) was noted on plot basis and then converted into $\mathrm{kg} \mathrm{ha}^{-1}$. To compute total biological yield, noted weight was added to the already calculated achene yield $\left(\mathrm{kg} \mathrm{ha}^{-1}\right)$.

\section{Plant water relations}

Water potential, relative leaf water content, turgor pressure and osmotic potential were recorded 10 days after the ABA foliar application. To find the relative leaf water content (RLWC), third leaf from the top (fully expanded) of two different plants from different treatment was taken. Leaves were cut at base of lamina and wrapped in plastic bags then transported to laboratory immediately. Fresh weight (FW) was calculated after two hours. After that turgid weight (TW) was found by saturating leaves in distilled water for time period of 16-18 $\mathrm{h}$ at room temperature. After saturation, leaves were prudently and rapidly blotted dry to find turgid weight. Dry weight (DW) was calculated after drying of leaf samples in oven for time period $72 \mathrm{~h}$ at $70{ }^{\circ} \mathrm{C}$. Relative leaf water content (RLWC) was calculated through the procedure of Schonfeld et al., 1988 and then averaged (Eq. 1).

$$
R L W C(\%)=(F W-D W) /(T W-D W) \times 100
$$

where, FW= fresh weight of leaf; DW= dry weight of leaf; and TW = turgid weight of leaf.

Leaf water potential was ranged from 8.00 to 10.00 A.M. It was computed through Scholander pressure chamber by following the method of Scholander et al. (1965). In order to compute, water potential, leaf was kept in a freezer at $-20{ }^{\circ} \mathrm{C}$ for 7 days. After that frozen leaf was defrosted in order to extract cell sap through a disposable syringe. Then osmotic potential was computed from extracted sap via an osmometer (Wescor $5500)$. Turgor pressure was calculated from the difference of water potential $\left(\Psi_{w}\right)$ and osmotic potential ( $\Psi \mathrm{s})(E q .2)$.

$$
\left(\Psi_{p}\right)=\left(\Psi_{w}\right)-\left(\Psi_{s}\right)
$$

\section{Quality parameters}

\section{Oil content}

Oil content in seeds was calculated using Soxhlet Fat Extraction method (AOAC, 1990). For about $10 \mathrm{~h}$, seeds were dried in an oven at $105^{\circ} \mathrm{C}$. Seeds were weighed before and after drying for determination of moisture content. For oil content determination, two grams of achenes per thimble were crushed in a coffee mill. Thimbles were weighed individually, crushed seeds were added and the final weight was estimated. Later, the thimbles were placed in extractors. Six dry and clean round bottom $250 \mathrm{ml}$ flasks were weighed and their weight noted. Solvent (petroleum ether) was added to flasks, attached to the extractors and placed on heating mantles attached to with condensers. Flasks were heated and extraction was continued for at least $6 \mathrm{~h}$, 
stopped extraction, removed thimbles and then reheated the flasks, so that all of the solvent might be collected in the Soxhlet extractors. The apparatus permitted to cool and flasks dried at $105{ }^{\circ} \mathrm{C}$ for $1 \mathrm{~h}$. After cooling, the flasks and oil were weighed together. Percent oil content was calculated via the following equation (Eq. 3).

$$
\text { Oil contents }(\%)=\frac{(\text { flask weight }+ \text { oil weight })-\text { flask weight }}{(\text { flask weight }+ \text { seed weight })-\text { flask weight }} \times 100
$$

\section{Fatty acid profile}

Gas liquid chromatography was used to determine fatty acid composition in sunflower oil by following Martin (1979). Oil was extracted from seed of Helianthus annuus hybrids by using Rancy oil seed crusher. In a loop of oil, $0.5 \mathrm{ml}$ petroleum and $1 \mathrm{ml}$ methylating solution ether was added in test tube. Solution was swirled to disperse loop and petroleum ether was also added to rinse loop during sampling. After addition of $1 \mathrm{ml}$ distilled water; it was kept for $10 \mathrm{~min}$. One $\mu 1$ solution was taken from upper layer and injected into the gas chromatograph. The total peak area and area of each fatty acid peak was computed by an electronic integrator and expressed as percentage of the total area of the peak.

\section{Statistical analysis}

Collected data was analyzed by using Fisher's analysis of variance technique in MSTAT-C. The differences among treatments, means etc. was compared through least significant difference (LSD) test at 5\% probability (Steel et al., 1997). Factorial experiment under Randomized Complete Block Design was used for analysis over years. Microsoft Excel Program was used for contrast study (Microsoft, version 2013).

\section{Results}

\section{Response of agronomic traits of sunflower to irrigation schedules and $A B A$ application}

Effect of year on plant height was statistically found non-significant during 2016 and 2017 but taller plants were observed in 2017 over 2016 (Table 2). Irrigation levels and ABA application exhibited significant impact on plant height during 2016-17 while their interactions were found non-significant. Under no water stress, plant height was significantly enhanced. Water deficit significantly reduced plant height with a larger reduction in plant height when stress was enforced at vegetative over at flowering stage. Negative impact of water deficit at both stages might be mitigated by spraying of ABA. In different $\mathrm{ABA}$ concentrations and its application stages, $10 \mu \mathrm{M} \mathrm{ABA}$ spray under water deficit at vegetative or at flowering stage considerably maximized plant height (Table 2). Orthogonal contrasts showed that 4 irrigations produced taller plants over three irrigations; ABA applied at vegetative stage produced taller plants over when applied at flowering stage. The contrast between $10 \mu \mathrm{M}$ and $20 \mu \mathrm{M}$ ABA was nonsignificant (Table 2).

Effect of year on head diameter was statistically not-significant during 2016 and 2017 but larger head diameter was observed in 2017 over 2016. Head diameter was significantly influenced by irrigation schedules and foliar spray of ABA at diverse growth stages while interactive effect had non-significant impact during both years of 
study (Table 2). Water deficit reduced head diameter over no stress. Water stress imposed at flowering stage caused significant reduction in head diameter and this reduction was more prominent in those treatments where stress was imposed at vegetative stage. Negative impact of water stress at both stages might be declined by foliar spray of ABA (Table 2). Orthogonal contrasts showed that 4 irrigations vs. 3 irrigations, ABA application at vegetative stage vs. ABA application at flowering and 3 irrigations (miss at vegetative) vs. 3 irrigations (miss at flowering) were significant but orthogonal contrast $10 \mu \mathrm{M}$ ABA vs. $20 \mu \mathrm{M}$ ABA was statistically non-significant during 2016-17.

Table 2. Agronomic traits of sunflower as affected by irrigation levels and ABA application

\begin{tabular}{|c|c|c|c|c|c|c|c|c|}
\hline \multirow{2}{*}{$\begin{array}{l}\text { Irrigation } \\
\text { levels (I) }\end{array}$} & \multicolumn{2}{|c|}{$\begin{array}{c}\text { Plant height } \\
(\mathbf{c m})\end{array}$} & \multicolumn{2}{|c|}{$\begin{array}{l}\text { Head diameter } \\
(\mathrm{cm})\end{array}$} & \multicolumn{2}{|c|}{$\begin{array}{c}\text { Achene yield } \\
\left(\mathrm{kg} \mathrm{ha}^{-1}\right)\end{array}$} & \multicolumn{2}{|c|}{$\begin{array}{c}\text { Biological yield } \\
\left(\mathrm{kg} \mathrm{ha}^{-1}\right)\end{array}$} \\
\hline & 2016 & 2017 & 2016 & 2017 & 2016 & 2017 & 2016 & 2017 \\
\hline $\mathrm{I}_{1}$ & $190.99 a$ & $193.31 \mathrm{a}$ & $16.87 \mathrm{a}$ & $17.57 \mathrm{a}$ & $2696.98 \mathrm{a}$ & $2859.33 \mathrm{a}$ & $12133.37 \mathrm{a}$ & $12322.65 a$ \\
\hline $\mathrm{I}_{2}$ & $170.71 \mathrm{c}$ & $171.02 \mathrm{c}$ & $12.60 \mathrm{~b}$ & $12.73 b$ & $2043.77 b$ & $2192.47 b$ & $11744.09 b$ & $11890.12 b$ \\
\hline $\mathrm{I}_{3}$ & $180.90 \mathrm{~b}$ & $182.81 \mathrm{~b}$ & $10.41 \mathrm{c}$ & $10.78 \mathrm{c}$ & $1951.31 \mathrm{c}$ & $2002.18 \mathrm{c}$ & $11240.79 \mathrm{c}$ & $11128.35 \mathrm{c}$ \\
\hline LSD (0.05) & 1.09 & 1.2 & 0.28 & 0.37 & 39.77 & 41.49 & 25.48 & 24.98 \\
\hline \multicolumn{9}{|c|}{ ABA levels and its application stages (C) } \\
\hline $\mathrm{C}_{1}$ & $182.78 \mathrm{a}$ & $183.47 \mathrm{a}$ & $13.93 \mathrm{a}$ & $14.15 \mathrm{a}$ & $2252.87 b$ & $2387.68 \mathrm{a}$ & $12028.15 \mathrm{a}$ & $11990.02 \mathrm{c}$ \\
\hline $\mathrm{C}_{2}$ & $180.60 \mathrm{~b}$ & $183.20 \mathrm{a}$ & $13.90 \mathrm{a}$ & $14.57 \mathrm{a}$ & 2359.91a & $2483.96 \mathrm{a}$ & $11900.68 b$ & $12010.88 b$ \\
\hline $\mathrm{C}_{3}$ & $183.49 \mathrm{a}$ & $183.43 \mathrm{a}$ & $13.89 \mathrm{a}$ & $14.24 \mathrm{a}$ & $2359.89 a$ & $2457.96 \mathrm{a}$ & $12023.25 \mathrm{a}$ & $12193.92 \mathrm{a}$ \\
\hline $\mathrm{C}_{4}$ & $178.04 \mathrm{bc}$ & $179.61 \mathrm{~b}$ & $12.62 b$ & $12.94 b$ & $2111.47 \mathrm{c}$ & $2259.34 b$ & $11289.99 \mathrm{c}$ & $11467.38 \mathrm{~d}$ \\
\hline $\mathrm{C}_{5}$ & $179.40 \mathrm{~b}$ & $181.11 \mathrm{~b}$ & $12.10 \mathrm{c}$ & $12.56 \mathrm{~b}$ & $2069.23 \mathrm{~d}$ & $2169.17 \mathrm{c}$ & $11288.34 \mathrm{c}$ & $11239.66 \mathrm{e}$ \\
\hline $\operatorname{LSD}(0.05)$ & 1.41 & 1.55 & 0.37 & 0.48 & 51.34 & 53.63 & 32.89 & 32.25 \\
\hline Interaction & $\mathrm{ns}$ & ns & $\mathrm{ns}$ & $\mathrm{ns}$ & ns & ns & ns & ns \\
\hline
\end{tabular}

Mean values in column carrying different letters are statistically significant at $5 \%$ probability level. $\mathrm{I}_{1}$ : No stress (4 irrigations), $\mathrm{I}_{2}$ : Stress at vegetative stage (3 irrigations), $\mathrm{I}_{3}$ : Stress at flowering stage (3 irrigations), $\mathrm{C}_{1}$ : Control, $\mathrm{C}_{2}: 10 \mu \mathrm{M} \mathrm{ABA}$ at vegetative stage, $\mathrm{C}_{3}: 10 \mu \mathrm{M} \mathrm{ABA}$ at flowering stage, $\mathrm{C}_{4}$ : $20 \mu \mathrm{M}$ ABA at vegetative stage, $\mathrm{C}_{5}: 20 \mu \mathrm{M}$ ABA at flowering stage, ns: non-significant

Table 2a. Orthogonal contrasts of agronomic traits

\begin{tabular}{|c|c|c|c|c|c|c|c|c|}
\hline \multirow[t]{2}{*}{ Orthogonal contrasts } & \multicolumn{2}{|c|}{$\begin{array}{c}\text { Plant height } \\
(\mathrm{cm})\end{array}$} & \multicolumn{2}{|c|}{$\begin{array}{l}\text { Head diameter } \\
(\mathrm{cm})\end{array}$} & \multicolumn{2}{|c|}{$\begin{array}{c}\text { Achene yield } \\
\left(\mathrm{kg} \mathrm{ha}^{-1}\right)\end{array}$} & \multicolumn{2}{|c|}{$\begin{array}{l}\text { Biological } \\
\text { yield }\left(\mathrm{kg} \mathrm{ha}^{-1}\right)\end{array}$} \\
\hline & 2016 & 2017 & 2016 & 2017 & 2016 & 2017 & 2016 & 2017 \\
\hline 4 irrigations vs. 3 irrigations & $*$ & $*$ & $*$ & $*$ & $*$ & $*$ & $*$ & $*$ \\
\hline $10 \mathrm{~m} \mathrm{ABA}$ vs. $20 \mathrm{~m} \mathrm{ABA}$ & ns & $\mathrm{ns}$ & $\mathrm{ns}$ & $\mathrm{ns}$ & ns & $\mathrm{ns}$ & ns & $\mathrm{ns}$ \\
\hline $\begin{array}{c}\text { ABA spray at vegetative vs. ABA spray at } \\
\text { flowering stage }\end{array}$ & * & * & * & * & * & $*$ & * & $*$ \\
\hline $\begin{array}{l}3 \text { irrigations (missed at vegetative stage) } \\
\text { vs. } 3 \text { irrigations (missed at flowering stage) }\end{array}$ & $*$ & * & * & $*$ & * & * & * & $*$ \\
\hline
\end{tabular}

*: Significant, ns: non-significant

Effect of year was statistically significant for achene yield during both years and significantly maximum achene yield was observed in 2017 over 2016 (Table 2). 
Analysis of the data exhibited that achene yield was significantly influenced by irrigation levels and spray of ABA at diverse growth stages while interactive impact had not-significant impact during both years (Table 2). Water stress reduced achene yield over no stress. When drought was enforced at flowering stage achene yield was significantly reduced and this reduction was more over stress imposed at vegetative stage. Damaging impacts of water stress at both stages might be decreased by foliar ABA spray (Table 2). Orthogonal contrasts for 4 irrigations vs. 3 irrigations, ABA application at vegetative stage vs. ABA application at flowering stage and 3 irrigations (miss at vegetative) vs. 3 irrigations (miss at flowering) were significant but orthogonal contrast $10 \mu \mathrm{M}$ ABA vs. $20 \mu \mathrm{M}$ ABA was statistically non-significant during 2016-17.

Year impact on biological yield was statistically significant during 2016-17 and significantly maximum biological yield was observed in 2017 over 2016. Irrigation levels and ABA spray significantly affected biological yield while their interaction was found insignificant. Biological yield was significantly increased in control treatments (no water stress) over stress imposed at vegetative or flowering stage (Table 2). Under various irrigation schedules influence of ABA application was deviating (Table 2). When the crop encountered no water stress, foliar spray of 10 or $20 \mu \mathrm{M} \mathrm{ABA}$ at vegetative or flowering stage considerably reduced biological yield with respect to that of the control during 2016-17. Orthogonal contrasts between 4 irrigations vs. 3 irrigations, $\mathrm{ABA}$ application at vegetative vs. $\mathrm{ABA}$ application at flowering 3 irrigations (miss at vegetative) vs. 3 irrigations (miss at flowering) were significant for biological yield whereas orthogonal contrast $10 \mu \mathrm{M}$ ABA vs. $20 \mu \mathrm{M}$ ABA was statistically nonsignificant during both years.

\section{Response of plant water relations to irrigation schedules and $A B A$ application}

Effect of year on leaf water potential was non-significant during 2016 and 2017 and leaf water potential was maximum in 2017 over 2016. Levels of irrigation and foliar ABA spray effect was prominent on leaf water potential during both years. Water deficit significantly reduced leaf water potential over control. Leaf water potential was significantly reduced when water stress imposed at flowering over vegetative stage. Mean values clearly showed that foliar ABA application significantly influenced the leaf water potential (Table 3). When crop encountered water deficit at vegetative stage, the application of $10 \mu \mathrm{M}$ ABA significantly improved leaf water potential with respect to control but it was statistically comparable with $20 \mu \mathrm{M}$ ABA spray at vegetative stage (Table 3). Orthogonal contrasts for 4 irrigations vs. 3 irrigations, ABA application at vegetative vs. ABA application at flowering and 3 irrigations (miss at vegetative stage) vs. 3 irrigations (miss at flowering) were significant but the contrast $10 \mu \mathrm{M}$ ABA vs. $20 \mu \mathrm{M}$ ABA was statistically non-significant during 2016-17.

Year impact on leaf osmotic potential was statistically significant during 2016-17 and significantly maximum leaf osmotic potential was observed in 2016 over 2017. Irrigation schedules and ABA application significantly influenced leaf osmotic potential while their interactions during both years were found insignificant. Water stress at both vegetative and flowering stage significantly reduced leaf osmotic potential over control. Leaf osmotic potential was reduced when water stress executed at flowering over vegetative stage. Mean values showed that foliar application of ABA significantly influenced the leaf osmotic potential. Under diverse irrigation schedules, performance of foliar application of ABA was divergent. When crop encountered no water stress at vegetative or flowering stage, foliar $\mathrm{ABA}$ spray reduced leaf osmotic potential during 
2016-17. Orthogonal contrast between 4 irrigations vs. 3 irrigations and 3 irrigations (miss at vegetative stage) vs. 3 irrigations (miss at flowering stage) for leaf osmotic potential were significant during 2016 and 2017 but orthogonal contrast for ABA application at vegetative stage vs. ABA application at flowering stage was nonsignificant in 2016 and statistically significant in 2017 . Likewise contrast $10 \mu \mathrm{M}$ ABA vs. $20 \mu \mathrm{M}$ ABA was statistically non-significant during 2016-17.

Effect of year on leaf turgor pressure was statistically significant during 2016 and 2017 and statistically higher leaf turgor pressure was observed in 2017 over 2016. Leaf turgor pressure was significantly influenced by irrigation schedules and foliar application of $\mathrm{ABA}$ at various growth stages while their interaction had non-significant impact during 2016-17. Water deficit reduced leaf turgor pressure over no stress (Table 3). Turgor pressure was significantly reduced when water stress was enforced at flowering stage and this reduction was higher over when stress was enforced at vegetative stage (Table 2).

Application of $10 \mu \mathrm{M}$ ABA under water deficit at vegetative stage improved leaf turgor pressure with respect to control. Water deficit at vegetative stage and application of $20 \mu \mathrm{M}$ ABA enhanced turgor pressure than control and this enhancement was statistically comparable with $10 \mu \mathrm{M}$ ABA application at vegetative stage. When crop confronted water deficit at vegetative stage but ABA $(10$ or $20 \mu \mathrm{M})$ was sprayed at flowering stage, it considerably reduced turgor pressure over control. Comparable observation was noted during 2016-17. Orthogonal contrasts between 4 irrigations vs. 3 irrigations, ABA application at vegetative stage vs. ABA application at flowering stage and 3 irrigations (miss at vegetative) vs. 3 irrigations (miss at flowering stage) were significant for turgor pressure but contrast $10 \mu \mathrm{M}$ ABA vs. $20 \mu \mathrm{M}$ ABA was statistically non-significant during both 2016-17 (Table 3).

Table 3. Plant water relations of sunflower as affected by irrigation levels and $A B A$ application

\begin{tabular}{|c|c|c|c|c|c|c|c|c|}
\hline \multirow{2}{*}{$\begin{array}{l}\text { Irrigation } \\
\text { levels }\end{array}$} & \multicolumn{2}{|c|}{$\begin{array}{c}\text { Water potential } \\
\text { (-MPa) }\end{array}$} & \multicolumn{2}{|c|}{$\begin{array}{c}\text { Osmotic potential } \\
\text { (-MPa) }\end{array}$} & \multicolumn{2}{|c|}{$\begin{array}{c}\text { Turgor potential } \\
\text { (MPa) }\end{array}$} & \multicolumn{2}{|c|}{$\begin{array}{c}\text { Relative water content } \\
(\%)\end{array}$} \\
\hline & 2016 & 2017 & 2016 & 2017 & 2016 & 2017 & 2016 & 2017 \\
\hline $\mathrm{I}_{1}$ & $0.674 \mathrm{a}$ & $0.681 \mathrm{a}$ & $1.545 \mathrm{a}$ & $1.561 \mathrm{a}$ & $0.972 a$ & $0.881 \mathrm{a}$ & $85.57 \mathrm{a}$ & $86.36 \mathrm{a}$ \\
\hline $\mathrm{I}_{2}$ & $0.939 b$ & $0.949 b$ & $1.678 \mathrm{~b}$ & $1.696 \mathrm{~b}$ & $0.741 \mathrm{~b}$ & $0.748 b$ & $79.23 b$ & $79.76 b$ \\
\hline $\mathrm{I}_{3}$ & $1.042 \mathrm{c}$ & $1.053 \mathrm{c}$ & $1.704 \mathrm{c}$ & $1.723 \mathrm{c}$ & $0.664 \mathrm{c}$ & $0.671 \mathrm{c}$ & $75.82 \mathrm{c}$ & $76.81 \mathrm{c}$ \\
\hline $\operatorname{LSD}(0.05)$ & 0.015 & 0.015 & 0.019 & 0.021 & 0.11 & 0.014 & 0.28 & 0.43 \\
\hline \multicolumn{9}{|c|}{ ABA levels and its application stages } \\
\hline $\mathrm{C}_{1}$ & $0.829 \mathrm{a}$ & $0.838 \mathrm{a}$ & $1.608 \mathrm{~b}$ & $1.625 b$ & $0.779 \mathrm{a}$ & $0.798 \mathrm{a}$ & $80.33 b$ & $81.25 \mathrm{a}$ \\
\hline $\mathrm{C}_{2}$ & $0.831 \mathrm{a}$ & $0.840 \mathrm{a}$ & $1.596 \mathrm{a}$ & $1.613 \mathrm{a}$ & $0.766 \mathrm{~b}$ & $0.774 \mathrm{~b}$ & $80.92 \mathrm{a}$ & $81.68 \mathrm{a}$ \\
\hline $\mathrm{C}_{3}$ & $0.847 \mathrm{a}$ & $0.856 a$ & $1.626 \mathrm{c}$ & $1.643 \mathrm{c}$ & $0.780 \mathrm{a}$ & $0.788 \mathrm{a}$ & $80.45 b$ & $81.13 \mathrm{a}$ \\
\hline $\mathrm{C}_{4}$ & $0.965 b$ & $0.975 b$ & $1.694 \mathrm{~d}$ & $1.712 \mathrm{~d}$ & $0.726 \mathrm{c}$ & $0.737 \mathrm{c}$ & $79.99 \mathrm{c}$ & $80.91 \mathrm{ab}$ \\
\hline $\mathrm{C}_{5}$ & $0.951 b$ & $0.961 b$ & $1.687 \mathrm{~d}$ & $1.705 \mathrm{~d}$ & $0.737 \mathrm{c}$ & $0.744 \mathrm{c}$ & $79.30 \mathrm{~d}$ & $80.19 b$ \\
\hline $\operatorname{LSD}(0.05)$ & 0.019 & 0.019 & 0.012 & 0.012 & 0.014 & 0.014 & 0.36 & 0.563 \\
\hline Interaction & $\mathrm{ns}$ & ns & ns & ns & ns & $\mathrm{ns}$ & ns & ns \\
\hline
\end{tabular}

Mean values in column carrying different letters are statistically significant at $5 \%$ probability level. $\mathrm{I}_{1}$ : No stress (4 irrigations), $\mathrm{I}_{2}$ : Stress at vegetative stage (3 irrigations), $\mathrm{I}_{3}$ : Stress at flowering stage (3 irrigations), $\mathrm{C}_{1}$ : Control, $\mathrm{C}_{2}: 10 \mu \mathrm{M} \mathrm{ABA}$ at vegetative stage, $\mathrm{C}_{3}: 10 \mu \mathrm{M} \mathrm{ABA}$ at flowering stage, $\mathrm{C}_{4}$ : $20 \mu \mathrm{M}$ ABA at vegetative stage, $\mathrm{C}_{5}: 20 \mu \mathrm{M}$ ABA at flowering stage, ns: non-significant 
Table 3a. Orthogonal contrasts of plant water relations

\begin{tabular}{|c|c|c|c|c|c|c|c|c|}
\hline \multirow[t]{2}{*}{ Orthogonal contrasts } & \multicolumn{2}{|c|}{$\begin{array}{l}\text { Water potential } \\
\text { (-MPa) }\end{array}$} & \multicolumn{2}{|c|}{$\begin{array}{l}\text { Osmotic } \\
\text { potential } \\
(-\mathrm{MPa})\end{array}$} & \multicolumn{2}{|c|}{$\begin{array}{l}\text { Turgor } \\
\text { potential } \\
\text { (MPa) }\end{array}$} & \multicolumn{2}{|c|}{$\begin{array}{l}\text { Relative water } \\
\text { content }(\%)\end{array}$} \\
\hline & 2016 & 2017 & 2016 & 2017 & 2016 & 2017 & 2016 & 2017 \\
\hline 4 irrigations vs. 3 irrigations & $*$ & $*$ & $*$ & $*$ & $*$ & $*$ & $*$ & $*$ \\
\hline $10 \mathrm{~m} \mathrm{ABA}$ vs. $20 \mathrm{~m} \mathrm{ABA}$ & ns & $\mathrm{ns}$ & $\mathrm{ns}$ & ns & $\mathrm{ns}$ & $\mathrm{ns}$ & ns & $\mathrm{ns}$ \\
\hline $\begin{array}{c}\text { ABA spray at vegetative vs. ABA spray } \\
\text { at flowering stage }\end{array}$ & $*$ & $*$ & ns & $*$ & $*$ & $*$ & $*$ & $*$ \\
\hline $\begin{array}{c}3 \text { irrigations (missed at vegetative stage) } \\
\text { vs. } 3 \text { irrigations (missed at flowering } \\
\text { stage) }\end{array}$ & $*$ & $*$ & $*$ & $*$ & * & $*$ & $*$ & * \\
\hline
\end{tabular}

*: Significant, ns: non-significant

Effect of year on relative leaf water content was statistically not-significant during 2016-17 but relative leaf water content was slightly higher during 2017 over 2016. Analysis of the data revealed that relative leaf water content was significantly influenced by irrigation schedules and foliar application of ABA at diverse growth stages while their interaction had non-significant impact during both years (Table 3). Water deficit reduced relative leaf water content over no stress. When water deficit was imposed at flowering stage relative leaf water content was significantly reduced and this reduction was higher over where stress was enforced at vegetative stage. Negative impact of water deficit at both stages might be decreased by foliar spray of ABA (Table 3). Influence of foliarly applied ABA was different under diverse irrigation levels (Table 3). No water deficit at both growth stages, foliar application of ABA reduced relative leaf water content during 2016-17. Orthogonal contrasts between 4 irrigations vs. 3 irrigations, ABA application at vegetative stage vs. ABA application at flowering stage and 3 irrigations (miss at vegetative stage) vs. 3 irrigations (miss at flowering stage) were significant for relative leaf water content but orthogonal contrast for $10 \mu \mathrm{M}$ ABA vs. $20 \mu \mathrm{M}$ ABA was statistically no-significant during 2016-17.

\section{Response of quality parameters of sunflower to irrigation schedules and $A B A$ application}

Effect of year on achene oil content was statistically non-significant but oil content was higher in 2017 with respect to 2016 (Table 4). Mean values exhibited that oil content was significant during irrigation treatments and foliar application of $\mathrm{ABA}$ at various growth stages while their interactions was found non-significant during both years (Table 4). Water stress reduced oil content compared to no stress. Oil content significantly reduced when water deficit was executed at flowering stage and this reduction was higher over when deficit was enforced at vegetative stage (Table 4). Under water deficit condition foliar spray of $10 \mu \mathrm{M}$ ABA at vegetative stage improved oil content over control. Water deficit at vegetative stage and $20 \mu \mathrm{M}$ ABA application also enhanced oil content than control but this enhancement was statistically comparable with $10 \mu \mathrm{M}$ ABA application at vegetative stage. When crop confronted water deficit at vegetative stage but ABA $(10$ or $20 \mu \mathrm{M})$ was sprayed at flowering stage, it significantly reduced oil content than control. Comparable observation was observed during 2016-17. 
Impact of year on stearic acid content was statistically significant during 2016-17 and maximum stearic acid content was observed during 2016 over 2017. Analysis of the data revealed that irrigation levels and ABA application exhibited significant impact on stearic acid content during 2016-17. Water deficit significantly improved stearic acid content and maximum increment in stearic acid content was noted when water deficit was executed at flowering stage over at vegetative stage (Table 4). Foliar spray of $20 \mu \mathrm{M} \mathrm{ABA}$ at flowering stage by enforcing stress at vegetative stage significantly enhanced stearic acid content over all treatments. Whereas spraying 10 or $20 \mu \mathrm{M}$ ABA at vegetative stage under water stress at vegetative stage reduced stearic acid content than control. Similar trend was noticed during 2016-17. Orthogonal contrasts between 4 irrigations vs. 3 irrigations and 3 irrigations (miss at vegetative stage) vs. 3 irrigations (miss at flowering stage) were significant for stearic acid content but orthogonal contrasts $10 \mu \mathrm{M}$ ABA vs. $20 \mu \mathrm{M}$ ABA, and $\mathrm{ABA}$ application at vegetative stage vs. ABA application at flowering stage were statistically non-significant during 2016-17.

Orthogonal contrasts for 4 irrigations vs. 3 irrigations, ABA application at vegetative stage vs. ABA application at flowering stage and 3 irrigations (miss at vegetative stage) vs. 3 irrigations (miss at flowering stage) were significant for oil content but orthogonal contrast $10 \mu \mathrm{M}$ ABA vs. $20 \mu \mathrm{M}$ ABA was statistically non-significant during 2016-17.

Effect of year on oleic acid content was statistically significant during 2016 and 2017 and statistically higher oleic acid was observed in 2016 over 2017. Oleic acid content was statistically influenced by irrigation levels and ABA spray while their interaction was found insignificant. Water stress significantly improved oleic acid content and maximum increment in oleic acid content was noted when water deficit was executed at vegetative stage over at flowering stage (Table 4). Water stress at vegetative stage and spraying of $20 \mu \mathrm{M}$ ABA at flowering stage had significantly improved oleic acid content over all treatments. Enforcing water deficit at vegetative stage and spraying $10 \mu \mathrm{M} \mathrm{ABA}$ at flowering stage also significantly improved oleic acid content with respect to control but this increment was lower with respect to spraying of $20 \mu \mathrm{M}$ ABA at the similar stage. Whereas spraying 10 or $20 \mu \mathrm{M}$ ABA at vegetative stage under water deficit at vegetative stage reduced oleic acid content than control. Same trend was observed during 2016-17. Orthogonal contrasts for 4 irrigations vs. 3 irrigations was significant but orthogonal contrasts $10 \mu \mathrm{M}$ ABA vs. $20 \mu \mathrm{M}$ ABA and ABA application at vegetative stage vs. ABA application at flowering stage and 3 irrigations (miss at vegetative stage) vs. 3 irrigations (miss at flowering stage) were statistically nonsignificant during both years.

Palmitic acid content was significantly affected during 2016 and 2017 and statistically higher palmitic acid content was observed in 2017 over 2016. Different irrigation levels and foliar spray of ABA at diverse growth stages significantly affected palmitic acid content while their interaction was found insignificant. Water stress reduced palmitic acid content over no stress. Palmitic acid content was considerably minimized when water stress was enforced at flowering stage and this reduction was maximum than where water deficit was enforced at vegetative stage (Table 4). Spray of $10 \mu \mathrm{M}$ ABA under water stress at vegetative stage statistically increased palmitic acid content over all treatments. Water stress at vegetative stage and application of $20 \mu \mathrm{M}$ ABA also enhanced palmitic acid content than control but this enhancement was lower than spraying of $10 \mu \mathrm{M} A B A$ at vegetative stage. When crop confronted water stress at vegetative stage but $\mathrm{ABA}(10$ or $20 \mu \mathrm{M})$ was sprayed at flowering stage, it significantly reduced palmitic acid content over control. Same results was noted during both years. 
Orthogonal contrasts between 4 irrigations vs. 3 irrigations was significant for palmitic acid content but orthogonal contrasts $10 \mu \mathrm{M}$ ABA vs. $20 \mu \mathrm{M}$ ABA, 3 irrigations (miss at vegetative stage) vs. 3 irrigations (miss at flowering stage) and ABA application at vegetative vs. ABA application at flowering stage were statistically non-significant during 2016-17.

Table 4. Oil content and fatty acid profile of sunflower as affected by irrigation levels and ABA application

\begin{tabular}{|c|c|c|c|c|c|c|c|c|c|c|}
\hline \multirow{2}{*}{$\begin{array}{l}\text { Irrigation levels } \\
\text { (I) }\end{array}$} & \multicolumn{2}{|c|}{$\begin{array}{c}\text { Oil content } \\
(\%)\end{array}$} & \multicolumn{2}{|c|}{$\begin{array}{c}\text { Stearic acid } \\
(\%)\end{array}$} & \multicolumn{2}{|c|}{$\begin{array}{c}\text { Oleic acid } \\
(\%)\end{array}$} & \multicolumn{2}{|c|}{$\begin{array}{c}\text { Palmitic acid } \\
(\%)\end{array}$} & \multicolumn{2}{|c|}{$\begin{array}{c}\text { Linoleic acid } \\
(\%)\end{array}$} \\
\hline & 2016 & 2017 & 2016 & 2017 & 2016 & 2017 & 2016 & 2017 & 2016 & 2017 \\
\hline $\mathrm{I}_{1}$ & $41.42 \mathrm{a}$ & $41.61 \mathrm{a}$ & $3.49 \mathrm{c}$ & $3.40 \mathrm{c}$ & $11.63 \mathrm{c}$ & $11.52 \mathrm{c}$ & $6.78 \mathrm{a}$ & $6.83 a$ & $78.31 \mathrm{a}$ & $78.37 \mathrm{a}$ \\
\hline $\mathrm{I}_{2}$ & $39.96 b$ & $40.34 b$ & $3.65 b$ & $3.55 b$ & $11.79 \mathrm{a}$ & $11.68 \mathrm{a}$ & $5.61 \mathrm{~b}$ & $5.769 \mathrm{~b}$ & $76.91 b$ & $76.85 b$ \\
\hline $\mathrm{I}_{3}$ & $39.28 \mathrm{c}$ & $39.46 \mathrm{c}$ & $3.79 \mathrm{a}$ & $3.66 \mathrm{a}$ & $11.72 b$ & $11.67 \mathrm{~b}$ & $5.36 \mathrm{c}$ & $5.50 \mathrm{c}$ & $76.55 \mathrm{c}$ & $76.65 c$ \\
\hline $\operatorname{LSD}(0.05)$ & 0.23 & 0.26 & 0.081 & 0.07 & 0.01 & 0.01 & 0.012 & 0.014 & 0.013 & 0.015 \\
\hline \multicolumn{11}{|c|}{ ABA levels and its application stages (C) } \\
\hline $\mathrm{C}_{1}$ & $40.49 \mathrm{a}$ & $40.88 \mathrm{a}$ & $3.61 \mathrm{c}$ & $3.51 \mathrm{c}$ & $11.88 \mathrm{c}$ & $11.61 \mathrm{c}$ & $5.97 \mathrm{a}$ & $6.06 \mathrm{a}$ & $77.27 \mathrm{~b}$ & $77.26 \mathrm{c}$ \\
\hline $\mathrm{C}_{2}$ & $40.46 \mathrm{a}$ & $40.70 \mathrm{a}$ & $3.61 \mathrm{c}$ & $3.47 \mathrm{~d}$ & $11.72 b$ & $11.65 b$ & $5.94 b$ & $6.04 \mathrm{~b}$ & $77.34 \mathrm{a}$ & $77.45 \mathrm{a}$ \\
\hline $\mathrm{C}_{3}$ & $40.18 b$ & $40.37 \mathrm{~b}$ & $3.62 \mathrm{c}$ & $3.52 \mathrm{bc}$ & $11.63 \mathrm{~d}$ & $11.53 \mathrm{~d}$ & $5.97 \mathrm{a}$ & $6.05 \mathrm{ab}$ & $77.22 \mathrm{~d}$ & $77.30 \mathrm{~b}$ \\
\hline $\mathrm{C}_{4}$ & $40.07 b$ & $40.25 b$ & $3.64 b$ & $3.53 b$ & $11.82 \mathrm{a}$ & $11.68 \mathrm{a}$ & $5.87 \mathrm{c}$ & $5.96 \mathrm{c}$ & $77.24 \mathrm{c}$ & $77.20 \mathrm{~b}$ \\
\hline $\mathrm{C}_{5}$ & $39.88 \mathrm{~b}$ & $40.13 b$ & $3.74 a$ & $3.62 \mathrm{a}$ & $11.73 \mathrm{~b}$ & $11.65 \mathrm{~b}$ & $5.81 \mathrm{~d}$ & $5.92 \mathrm{~d}$ & $77.18 \mathrm{e}$ & $77.23 \mathrm{~b}$ \\
\hline $\operatorname{LSD}(0.05)$ & 0.3 & 0.34 & 0.02 & 0.01 & 0.01 & 0.012 & 0.013 & 0.012 & 0.011 & 0.01 \\
\hline Interaction & $\mathrm{ns}$ & $\mathrm{ns}$ & $\mathrm{ns}$ & $\mathrm{ns}$ & $\mathrm{ns}$ & $\mathrm{ns}$ & $\mathrm{ns}$ & $\mathrm{ns}$ & $\mathrm{ns}$ & $\mathrm{ns}$ \\
\hline
\end{tabular}

Mean values in column carrying different letters are statistically significant at $5 \%$ probability level. $\mathrm{I}_{1}$ : No stress (4 irrigations), $\mathrm{I}_{2}$ : Stress at vegetative stage (3 irrigations), $\mathrm{I}_{3}$ : Stress at flowering stage (3 irrigations), $\mathrm{C}_{1}$ : Control, $\mathrm{C}_{2}: 10 \mu \mathrm{M} \mathrm{ABA}$ at vegetative stage, $\mathrm{C}_{3}$ : $10 \mu \mathrm{M} \mathrm{ABA}$ at flowering stage, $\mathrm{C}_{4}$ : $20 \mu \mathrm{M}$ ABA at vegetative stage, $\mathrm{C}_{5}: 20 \mu \mathrm{M}$ ABA at flowering stage, ns: non-significant

Table 4a. Orthogonal contrasts of oil content and fatty acid profile of sunflower

\begin{tabular}{|c|c|c|c|c|c|c|c|c|c|c|}
\hline \multirow[b]{2}{*}{ Orthogonal contrasts } & \multicolumn{2}{|c|}{$\begin{array}{c}\text { Oil } \\
\text { content } \\
(\%)\end{array}$} & \multicolumn{2}{|c|}{$\begin{array}{c}\text { Stearic } \\
\text { acid }(\%)\end{array}$} & \multicolumn{2}{|c|}{$\begin{array}{c}\text { Oleic } \\
\text { acid }(\%)\end{array}$} & \multicolumn{2}{|c|}{$\begin{array}{l}\text { Palmitic } \\
\text { acid }(\%)\end{array}$} & \multicolumn{2}{|c|}{$\begin{array}{l}\text { Linoleic } \\
\text { acid }(\%)\end{array}$} \\
\hline & $\begin{array}{c}201 \\
6\end{array}$ & $\begin{array}{c}201 \\
7\end{array}$ & $\begin{array}{c}201 \\
6\end{array}$ & $\begin{array}{c}201 \\
7\end{array}$ & $\begin{array}{c}201 \\
6\end{array}$ & $\begin{array}{c}201 \\
7\end{array}$ & 2016 & 2017 & 2016 & 2017 \\
\hline 4 irrigations vs. 3 irrigations & $*$ & $*$ & $*$ & $*$ & $*$ & $*$ & $*$ & $*$ & $*$ & $*$ \\
\hline $10 \mathrm{~m} \mathrm{ABA}$ vs. $20 \mathrm{~m} \mathrm{ABA}$ & ns & ns & ns & ns & $\mathrm{ns}$ & $\mathrm{ns}$ & $\mathrm{ns}$ & ns & ns & Ns \\
\hline $\begin{array}{c}\text { ABA spray at vegetative vs. ABA spray at } \\
\text { flowering stage }\end{array}$ & $*$ & $*$ & ns & ns & ns & ns & ns & ns & ns & Ns \\
\hline $\begin{array}{l}3 \text { irrigations (missed at vegetative stage) vs. } 3 \\
\text { irrigations (missed at flowering stage) }\end{array}$ & $*$ & $*$ & $*$ & $*$ & ns & ns & ns & $\mathrm{ns}$ & ns & Ns \\
\hline
\end{tabular}

*: Significant, ns: non-significant

Effect of year on linoleic acid content was significant and statistically higher linoleic acid was observed in 2017 over 2016. Linoleic acid content was significantly influenced by irrigation levels and foliar spray of ABA at various growth stages while their interaction was found non-significant effect on linoleic acid content. Water stress 
reduced linoleic acid content over no stress. Considerably lesser linoleic acid was noted when water stress was enforced at flowering stage than where water deficit was enforced at vegetative stage (Table 4). Spray of $10 \mu \mathrm{M}$ ABA under water stress at vegetative stage statistically augmented linoleic acid content over control. Water stress at vegetative stage and application of $20 \mu \mathrm{M}$ ABA also enhanced linoleic acid content than control but this enhancement was lower than spraying of $10 \mu \mathrm{M} A B A$ at vegetative stage. When crop confronted water deficit at vegetative stage but ABA (10 or $20 \mu \mathrm{M})$ was sprayed at flowering stage, it considerably reduced linoleic acid content over control. Same observation was recorded during 2016-17. Orthogonal contrasts for 4 irrigations vs. 3 irrigations was significant for linoleic acid content but orthogonal contrasts $10 \mu \mathrm{M}$ ABA vs. $20 \mu \mathrm{M}$ ABA and ABA application at vegetative stage vs. ABA application at flowering stage and 3 irrigations (miss at vegetative) vs. 3 irrigations (miss at flowering stage) were statistically non-significant during both years.

\section{Discussion}

\section{Agronomic and yield related traits}

Water stress is one of the severe threats to crop production in semi-arid and arid areas of Pakistan. In present trial, foliar spraying of abscisic acid d to sunflower under water deficit either at vegetative or flowering stage has been noticed to considerably enhanced crop growth, yield, osmotic adjustment, development, water relations, achene quality and fatty acid profile over no spray of ABA under water stress.

Water deficit both at vegetative or flowering stage declined sunflower plant height and maximum decrease in plant height was recorded when water deficit happened at bud initiation (Unger, 1983). Exogenous ABA application under no water stress condition reduced plant height. Under no water stress condition, shoot growth reduced by ABA application in maize seedling (Zhang et al., 2012) which finally reduced plant height. ABA foliar application under water stress enhanced plant height since it perform as shoot growth regulator under water scarce environments (Zhang et al., 2012). In the current trial less concentration $(10 \mu \mathrm{M}$ ABA) supported vegetative growth under water stress whereas the greater concentration $(20 \mu \mathrm{M}$ ABA) could have facilitated in survival of plant by regulating processes like plant size growth and stomatal opening (Sah et al., 2016). Controversially foliar spray of ABA under water stress and no stress condition decreased height of shoot in Populus davidiana which was owed to lesser accumulation of dry matter and its maximum distribution to Populus davidiana roots (Chunyang et al., 2004).

Water stress significantly reduced head diameter of sunflower and maximum reduction in head diameter happened when crop encountered water deficit at flowering over vegetative stage (Hussain et al., 2015, 2017). This decrease was due to reduction in photosynthates production (Dong et al., 2017; Mila et al., 2017) and their minimum distribution to the floral parts (Dong et al., 2017). Spraying of $10 \mu \mathrm{M}$ ABA under water deficit at flowering stage considerably enhanced head diameter because abscisic acid useful in water maintenance response of plants and may have enhanced plant growth through improvement in water use efficiency (WUE) (Wei et al., 2015; Dong et al., 2017).

For better production of crops, normal irrigations are essential but under water scarcity situation, it is significant to recognize important growth stage where irrigation can be missed without significantly reducing crop production (Wang et al. 2017). Water 
deficit both at vegetative or flower initiation stage reduced achene yield along with its attributes in Helianthus (Hussain et al., 2014, 2015). Harsh water scarcity effects were more apparent at flower initiation stage over at vegetative stage (Hussain et al., 2015). ABA foliar application during normal irrigation also considerably declined the yield, as it only enhanced yield during water deficit both at vegetative or flower initiation stage. From tolerance to dehydration, the plant hormone ABA played imperative (Wani et al., 2016) by helping dispersion of roots of maize in soil (Zhang et al., 2012) which finally augmented production of assimilates and yield through enhancing osmotic adjustment and water relation in plants like sunflower (Hussain et al., 2014, 2015; Wani et al., 2016; Dong et al., 2017). Abscisic acid sprayed under water deficit altered the sorghum physiology and growth and its application on seed enhanced grain yield (Traore and Sullivan, 1990). Contrary Ayub et al. (2000) described that foliar spray of abscisic acid during water stress to mung bean exhibited non-significant response to grain yield.

In current study biological yield of Helianthus was maximally reduced when water stress was enforced at vegetative over at flowering stage. Foliar application of 10 or $20 \mu \mathrm{M}$ ABA under water stress at vegetative stage enhanced biomass but maximum increment in biomass was observed when $10 \mu \mathrm{M}$ abscisic acid sprayed during this stage. This enhancement in biomass was due to decrease in shoot' water loss, roots deeper dispersion and increment in WUE which eventually augmented production of crop. Same findings were reported in Helianthus (Zhang et al., 2012; Hussain et al., 2015; Dong et al., 2017) and maize (Hartung et al., 1994). Contradictory ABA foliar application under water deficit and well-watered condition decreased biomass production in Populus which is owed to lesser dry matter accumulation and maximum distribution to roots of plant (Chunyang et al., 2004) ultimately may reduce above ground vegetation.

\section{Plant water relations}

Results of current trial showed that water potential became more adverse under water scarcity both at vegetative or at flowering stage and the exogenous application of abscisic acid considerably averted the negative impact of water stress by making the water potential in sunflower plants less negative. When ABA sprayed at vegetative stage over at flowering stage water potential became less negative. This enhancement in water potential indicate improvement of water shortage tolerance in helianthus by foliar application of abscisic acid, which eventually retained plant moisture through restricted and steady stomatal closure and by increasing root penetration and lastly help in plant survival through dehydrin protein production under cellular desiccation. Alfredo and Setter (2000) described that Cassava has capacity of partial stomatal closure under water stress due to abscisic acid accumulation in vegetative parts specifically in leaves which decreased leaf development and lastly continuation of growth happened after watering the crop again. Foliar application of abscisic acid responds to water stress condition by storing dehydrin protein in sunflower vegetative tissues (Aguado et al., 2014), poplar and wheat (Pelah et al., 1997). Dehydrin protein saves plants from cellular desiccation (Baker et al., 1988; Dure et al., 1989; Close, 1996). Jones and Corlett (1992) noticed contrary plant response as plant metabolic processes were more prone to cell size and turgor pressure compared to absolute water potential.

Water deficit both at vegetative or flowering stage depressed the water potential which also triggered a comparable reduction in osmotic potential. Ultimate decrease in osmotic potential was result of solutes accumulation in various plant parts through 
osmotic adjustments (Serraj and Sinclair, 2002; Hussain et al., 2015). ABA foliar application to helianthus caused osmotic potential less adverse. When ABA was sprayed at vegetative stage over at flowering stage, osmotic potential became less negative. This enhancement in osmotic potential was the result of compatible solutes accumulation like glycinebetaine (Serraj and Sinclair, 2002), proline (Hussain et al., 2015) and total soluble sugars. Such compounds are supportive in delay of desiccation through ROS detoxification, maintenance of membranes in addition to enzymes arrangement (Ludlow and Muchow, 1990; Subbarao et al., 2000). Hussain et al. (2015) exhibited that under water stress condition, key physiological mechanism was decrease in osmotic potential to keep leaf turgor pressure. Contrarily ABA foliar application may increment the action of definite enzymes such as ribonuclease and $\alpha$-amylase. These enzymes may be involved in breakdown of starches plus other related materials thus making osmotic potential more adverse (Dong et al., 2017; Mila et al., 2017).

Water stress at vegetative or flower initiation stage considerably decreased turgor pressure of leaf. Decrease in turgor pressure of leaf was owed to reduction in water potential and already reported in wheat (Blum, 1997) and Helianthus (Hussain et al., 2015). ABA foliar application during water stress considerably enhanced leaf turgor pressure which was maximum at vegetative over at flower initiation stage. This enhancement in leaf turgor potential was result of plant efforts to maintain moisture. These types of findings were also reported in maize (Zhang et al., 2012) and in poplar (Pelah et al., 1997).

Results of the current study exposed that water stress at vegetative or flowering stage severely decreased sunflower relative water content of leaf. Decline in relative leaf water content in Helianthus was also noticed by Hussain et al. (2015). Further reduction in relative water content of leaf was noticed when water deficit imposed at vegetative over flower initiation stage. ABA foliar application considerably improved relative leaf water content under water deficit situations. These enhancements in relative water content of leaf was owed to plant moisture preservation through limited and steady stomata closure, root proliferation for water abstraction and dehydrin protein buildup which ultimately rescued plant from cellular desiccation. The same result was noticed in poplar, wheat and cassava (Pelah et al., 1997; Alfredo and Setter, 2000; Aguado et al., 2014). Contrary to this, ABA foliar application to maize during water stress did not increment relative water content of leaf (Unyayar et al., 2004).

\section{Oil contents and fatty acid profile}

Water stress both at vegetative and flower initiation stage decreased achene oil content. Oil content was observed to increase when water deficit was applied at flower initiation stage over vegetative stage. Water deficit applied prior to reproductive stage (Saleem et al., 2013), at flower initiation stage (Hammadeh et al., 2005) and for the duration of seed filling stage (Mekki et al., 1999; Hussain et al., 2015) of Helianthus declined oil content. In contrast the seed oil content exhibited constancy during incrementing water scarcity (Khan et al., 2000). Hussain et al. (2015) also reported no significant reduction in oil content under water deficit. ABA spraying under water deficit at vegetative or at flower initiation stage statistically enhanced oil content. Seed oil content may be improved by abscisic acid foliar spray because its spray result in limited stomata closure, reduced transpiration rate, repressed shoot development and improved root proliferations (Alfredo and Setter, 2000; Saleem et al., 2013) which eventually improved water accessibility for oil production in achene yield. 
Water deficit and ABA foliar application under water deficit or well-watered conditions influenced fatty acid profile. Results of present trial emphasized that oleic acid and stearic acid reduced whereas linoleic acid and palmitic acid improved during well-watered and ABA foliar application improved oleic acid and stearic acid whereas reduced linoleic acid and palmitic acid. Water deficit at vegetative and at flower initiation stage slightly improved oleic acid and stearic acid whereas linoleic acid and palmitic acid reduced. Further increment was noticed in stearic acid when water deficit imposed at flower initiation stage whereas same response was noticed in oleic acid when sunflower encountered water deficit at vegetative stage. Linoleic acid and Palmitic acid were reduced under water shortage situation imposed at vegetative or at flower initiation stage but further decrease in both fatty acids was noted in crop that confronted water deficit at flower initiation stage. Flagella et al. (2002) also described reduction in oleic acid and stearic acid and increment in linoleic acid and palmitic acid in Helianthus under irrigation. Contrary, in Helianthus, oleic acid decreased and palmitic acid improved under water shortages (Saleem et al., 2013; Hussain et al., 2015). ABA foliar application both at vegetative and at flower initiation stage under water shortage somewhat reduced oleic acid and stearic acid, whereas its application to some extent enhanced palmitic acid and linoleic acid. This decrease in stearic and oleic acid and enhancement in linoleic and palmitic acid revealed that abscisic acid was useful in extenuating the negative impacts of water stress through enhancing water availability to plants. Water availability may be enhanced through maintaining moisture content in plant by restricted stomatal closure, decrease in transpiration rate, increment in root proliferation and inhibiting shoot growth (Alfredo and Setter, 2000; Hoad et al., 2001; Saleem et al., 2013; Hussain et al., 2015, 2017; Dong et al., 2017;).

\section{Conclusion}

It is concluded from this experiment that foliar spray of $10 \mu \mathrm{M}$ ABA under water sacristy at vegetative stage significantly enhanced plant height, head diameter, achene yield and biological yield with respect to no abscisic acid. It also enhanced water relations by enhancing turgor pressure and water potential which specifies that ABA foliar application was useful in improving sunflower drought tolerance.

Foliar application of $10 \mu \mathrm{M}$ abscisic acid at flowering stage by enforcing water stress at this stage also enhanced plant height, head diameter, achene yield and biological yield as compared to no ABA but this increment was lower than with the $10 \mu \mathrm{M} A B A$ foliar application at vegetative stage after enforcement of water deficit at this stage.

Acknowledgements. This work was supported by the National Key Research and Development Program of China (grant no. 2017YFC0504704) and the National Natural Science Foundation of China (51669034).

\section{REFERENCES}

[1] Aguado, A., Capote, N., Romero, F., Dodd, I. C., Colmenero-Flores, J. M. (2014): Physiological and gene expression responses of sunflower (Helianthus annuus L.) plants differ according to irrigation placement. - Plant Sci. 227: 37-44.

[2] Ahmad, W.; Noor, M.A.; Afzal, I.; Bakhtavar, M.A.; Nawaz, M.M.; Sun, X.; Zhou, B.; Ma, W.; Zhao, M. (2016): Improvement of sorghum crop through exogenous application of natural growth-promoting substances under a changing climate. - Sustainability. 8: 1330. 
[3] Alfredo, A. C. A., Setter, T. L. (2000): Response of cassava to water deficit: Leaf area growth and abscisic acid. - Crop Sci. 40: 131-137.

[4] AOAC (1990): Official Methods of Analysis.15th Ed. - Association of Official Analytical Chemists, Inc., Virginia, USA, pp. 770-771.

[5] Ayub, N., Bano, A., Ramzan, S., Usman, M. (2000): Effect of VAM on drought tolerance and growth of plant in comparison with the effect of growth regulators. - Pak. J. Biol. Sci. 3(6): 957-959.

[6] Baker, J., Steele, C., Dure, L. III. (1988): Sequence and characterization of 6 Lea protein and their genes from cotton. - Plant Mol. Biol.11: 277-291.

[7] Blum, A. (1997): Crop Responses to Drought and the Interpretation of Adaptation. - In: Belhassen, I. (ed.) Drought Tolerance in Higher Plants: Genetical, Physiological, and Molecular Biological Analysis. Kluwer Academic Publish, Dordrecht, pp. 57-70.

[8] Chunyang, L., Chunying, Y., Shirong, L. (2004): Different response of two contrasting Populus davidiana cultivars to exogenous abscisic acid application. - Environ. Exp. Bot. 51: 237-246.

[9] Close, T. J. (1996): Dehydrins: emergence of a biochemical role of a family of plant dehydration proteins. - Physiol. Plant. 97: 795-803.

[10] Daryanto, S., Wang, L., Jacinthe, P. A. (2016): Global synthesis of drought effects on maize and wheat production. - PloS one 11(5) e015(6362).

[11] Dong, B., Zheng, X., Liu, H., Able, J. A., Yang, H., Zhao, H., ... Liu, M. (2017): Effects of drought stress on pollen sterility, grain yield, abscisic acid and protective enzymes in two winter wheat cultivars. - Frontiers in Plant Science 8: 1008.

[12] Dure, L III, Crouch, M., Harada, J., Mundy, J., Quatrano, R., Thomas, T., Sung, Z. R. (1989): Common amino acid sequence domains among the LEA proteins of higher plants. - Plant Mol. Biol. 12: 475-486.

[13] Fernández, C., Alemano, S., Vigliocco, A., Andrade, A., Abdala, G. (2012): Stress Hormone Levels Associated with Drought Tolerance vs. Sensitivity in Sunflower (Helianthus annuus L.). - In: Khan, N. A. et al. (eds.) Phytohormones and Abiotic Stress Tolerance in Plants. Springer, Berlin Heidelberg, pp. 249-276.

[14] Finkelstein, R. (2013): Abscisic acid synthesis and response. - The Arabidopsis Book 11: e0166.

[15] Flagella, Z., Rotunno, T., Tarantino, E., Di Caterina, R., De Caro, A. (2002): Changes in seed yield and fatty acid composition of high oleic sunflower hybrids in relation to the sowing date and water regime. - Eur. J. Agron. 17: 221-230.

[16] Hammadeh, I., Maury, P., Debaeke, P., Lecoeur, J., Nouri, L., Kiani, S. P., Grieu, P. (2005): Canopy nitrogen distribution and photosynthesis during grain filling in irrigated and water stressed sunflower genotypes. - Interdrought-II, The Second International Conference on Integrated Approaches to Sustain and Improve Plant Production under Drought Stress, Rome, Italy. September, 24-28.

[17] Harris, J. (2015): Abscisic acid: hidden architect of root system structure. - Plants 4: 548572. DOI: $10.3390 /$ plants4030548.

[18] Hartung, W., Zhang, J., Davies, W. J. (1994): Does abscisic acid play a stress physiological role in maize plants growing in heavily compacted soil? - J. Exp. Bot. 45: 221-226.

[19] Hasanuzzaman, M., Nahar, K., Alam, M. M., Roychowdhury, R., Fujita, M. (2013): Physiological, biochemical, and molecular mechanisms of heat stress tolerance in plants. - International Journal of Molecular Sciences 14(5): 9643-9684.

[20] Hoad, S. P., Russell, G., Lucas, M. E., Bingham, I. J. (2001): The management of wheat, barley and oats root systems. - Adv. Agron. 74: 193-246.

[21] Hussain, M. M., Rauf, S., Riaz, M. A., Al-Khayri, J. M., Monneveux, P. (2017): Determination of drought tolerance related traits in Helianthus argophyllus, Helianthus annuus, and their hybrids. - Breeding Science 67(3): 257-267. http://doi.org/10.1270/jsbbs.16095. 
[22] Hussain, S., Saleem, M. F., Iqbal, J., Ibrahim, M., Atta, S., Ahmed, T., Rehmani, M. I. A. (2014): Exogenous application of abscisic acid may improve the growth and yield of sunflower hybrids under drought. - Pakistan Journal of Agricultural Sciences 51(1).

[23] Hussain, S., Saleem, M. F., Iqbal, J., Ibrahim, M., Ahmad, M., Nadeem, S. M., Ali, A., Atta, S. (2015): Abscisic acid mediated biochemical changes in sunflower (Helianthus annuus L.) grown under drought and well-watered field conditions. - Journal of Animal \& Plant Sciences 25(2): 406-416.

[24] Jones, H. G., Corlett, J. E. (1992): Current topics in drought physiology. - J. Agric. Sci. 119: 291296.

[25] Khan, M. S., Swati, M. S., Khali, I. H., Iqbal, A. (2000): Heterotic studies for various characters in sunflower. - Asian J. Plant Sci. 2(14): 1010-1014.

[26] Kim, D. Y., Jin, J. Y., Alejandro, S., Martinoia, E., Lee, Y. (2010): Overexpression of AtABCG36 improves drought and salt stress resistance in Arabidopsis. - Physiologia Plantarum 139(2): 170-180.

[27] Ludlow, M. M., Muchow, R. C. (1990): A critical evaluation of traits for improving crop yields in water-limited environments. - Adv. Agron. 43: 107-153.

[28] Martin, P. G. (1979): Manuals of Food Quality Control 3. Commodities. - Food and Agriculture Organization of the United Nations, Rome, pp. 313-320.

[29] Mehrotra, R., Bhalothia, P., Bansal, P., Basantani, M. K., Bharti, V., Mehrotra, S. (2014): Abscisic acid and abiotic stress tolerance. Different tiers of regulation. - Journal of Plant Physiology 171(7): 486-496.

[30] Mekki, B. B., El-Kholy, M. A., Mohamad, E. M. (1999): Yield, oil and fatty acids content as affected by water deficit and potassium fertilization in two sunflower cultivars. - Egyptian J. Agron. 35(2): 67-85.

[31] Mila, A. J., Ali, M. H., Akanda, A. R., Rashid, M. H. O., Rahman, M. A. (2017): Effects of deficit irrigation on yield, water productivity and economic return of sunflower. Cogent Food \& Agriculture 3(1): 1287619.

[32] Natsagdorj, E., Renchin, T., Kappas, M., Tseveen, B., Dari, C., Tsend, O., Duger, U. O. (2017): An integrated methodology for soil moisture analysis using multispectral data in Mongolia. - Geo-spatial Infor. Sci. 20: 46-55.

[33] Ng, L. M., Melcher, K., Teh, B. T., Xu, H. E. (2014): Abscisic acid perception and signaling: structural mechanisms and applications. - Acta Pharmacol. Sin. 35: 567-584.

[34] Pelah, D., Wang, W., Altman, A., Shoseyov, O., Bartels, D. (1997): Differential accumulation of water stress-related proteins, sucrose synthase and soluble sugars in Populus species that differ in their water stress response. - Physiol. Plant. 99: 153-159.

[35] Rauf, S., Al-Khayri, J. M., Zaharieva, M., Monneveux, P., Khalil, F. (2015): Breeding Strategies to Enhance Drought Tolerance in Crops. - In: Al-Khayri, J. M., Jain, S. M., Johnson, D. V. (eds.) Advances in Plant Breeding Strategies. Agronomic, Abiotic and Biotic Stress Traits. Springer, Switzerland.

[36] Sah, S. K., Reddy, K. R., Li, J. (2016): Abscisic acid and abiotic stress tolerance in crop plants. - Frontiers in Plant Science 7: 571.

[37] Saleem, A. H., Omar, A. E. A., Ali, M. M. A. (2013): Various responses of sunflower genotypes to water stress on newly reclaimed sandy soil. - Acta Agron. Hung. 61: 55-69.

[38] Saradadevi, R., Palta, J. A., Siddique, K. H. (2017): ABA-mediated stomatal response in regulating water use during the development of terminal drought in wheat. - Frontiers in Plant Science 8: 1251.

[39] Scholander, P. F., Hammel, H. T., Bradstreet, E. D., Hemmingsen, E. A. (1965): Sap pressure in vascular plants. - Science 148(3668): 339-346.

[40] Schonfeld, M. A., Johnson, R. C., Carver, B. F., Mornhinweg, D. W. (1988): Water relations in winter wheat as drought resistance indicator. - Crop Sci. 28: 526-531.

[41] Serraj, R., Sinclair, T. R. (2002): Osmolyte accumulation: can it really help increase crop yield under drought conditions? - Plant Cell Environ. 25: 333-341. 
[42] Song, X. P., Huang, C., Townshend, J. R. (2017): Improving global land cover characterization through data fusion. - Geo-spatial Infor. Sci. 20: 141-150.

[43] Sreenivasulu, N., Harshavardhan, V. T., Govind, G., Seiler, C., Kohli, A. (2012): Contrapuntal role of ABA: does it mediate stress tolerance or plant growth retardation under long-term drought stress? - Gene 506(2): 265-273.

[44] Steel, R. G. D., Torrie, J. H., Dicky, D. A. (1997): Principles and Procedures of Statistics: A Biometrical Approach. Third ed. - McGraw Hill Book Co. Inc. New York, pp. 400428.

[45] Subbarao, G. V., Num, N. H., Chauhan, Y. S., Johansen, C. (2000): Osmotic adjustment, water relation and carbohydrates remobilization in pigeonpea under water deficits. $-\mathrm{J}$. Plant Physiol. 157: 651-659.

[46] Traore, M., Sullivan, C. Y. (1990): Effect of abscisic acid treatment on sorghum drought responses. - Soc. Plant Physiol. Biochem. 2: 849-853.

[47] Tsironi, T. N., Taoukis, P. S. (2017): Effect of storage temperature and osmotic pretreatment with alternative solutes on the shelf-life of gilthead seabream (Sparus aurata) fillets. - Aquaculture and Fisheries 2: 39-47.

[48] Unger, P. W. (1983): Irrigation effects on sunflower growth, development and water use. - Field Crops Res. 3: 181-194.

[49] Unyayar, S., Keles, Y., Unal, E. (2004): Proline and ABA levels in two sunflower genotypes subjected to water stress. - Bulg. J. Plant Physiol. 30(3-4): 34-47.

[50] Villegas, G., Liao, W., Criollo, R., Philips, W., Ochoa, D. (2017): Detection of leaf structures in close-range hyperspectral images using morphological fusion. - Geo-spatial Infor. Sci. 20: 325-332.

[51] Wang, S., Wang, Z., Gu, J. (2017): Variation patterns of fine root biomass, production and turnover in Chinese forests. - J. Fores. Res. 28: 1185-1194.

[52] Wang, Z. L., Huang, B. R., Xu, Q. Z. (2003): Effects of abscisic acid on drought responses of Kentucky bluegrass. - J. Am. Soc. Hort. Sci. 128(1): 36-41.

[53] Wani, S. H., Kumar, V. (2015): Plant stress tolerance: engineering ABA: a potent phytohormone. - Transcriptomics 3: 113.

[54] Wani, S. H., Kumar, V., Shriram, V., Sah, S. K. (2016): Phytohormones and their metabolic engineering for abiotic stress tolerance in crop plants. - The Crop Journal 4(3): $162-176$.

[55] Wei, L., Wang, L., Yang, Y., Wang, P., Guo, T., Kang, G. (2015): Abscisic acid enhances tolerance of wheat seedlings to drought and regulates transcript levels of genes encoding ascorbate-glutathione biosynthesis. - Front. Plant Sci. 2015(6): 458.

[56] Woli, P., Jones, J. W., Ingram, K. T., Hoogenboom, G. (2014): Predicting crop yields with the agricultural reference index for drought. - Journal of Agronomy and Crop Science 200(3): 163-171.

[57] Wu, H., Chen, C., Wang, Q., Lin, J., Xue, J. (2017): The biological content of ballast water in China: a review. - Aquaculture and Fisheries 2: 241-246.

[58] Yin, D., Roderick, M. L., Leech, G., Sun, F., Huang, Y. (2014): The contribution of reduction in evaporative cooling to higher surface air temperatures during drought. Geophysical Research Letters 41(22): 7891-7897.

[59] Yin, Y., Gao, J., Liu, Y. (2017): Influence of industrial organizational structure on farming performance of large yellow croaker farmers. - Aquaculture and Fisheries 2: 134-139.

[60] Yoshida, T., Mogami, J., Yamaguchi-Shinozaki, K. (2014): ABA-dependent and ABAindependent signaling in response to osmotic stress in plants. - Current Opinion in Plant Biology 21: 133-139.

[61] Zhang, L., Gao, M., Hu, J., Zhang, X., Wang, K., Ashraf, M. (2012): Modulation role of abscisic acid (ABA) on growth, water relations and glycinebetaine metabolism in two maize (Zea mays L.) cultivars under drought stress. - International Journal of Molecular Sciences 13(3): 3189-3202. 